\title{
Correction to: Analysis of a Length-Structured Density-Dependent Model for Fish
}

\author{
Jason Callahan ${ }^{1} \cdot$ Eric Eager $^{2} \cdot$ Richard Rebarber $^{3} \cdot$ Eva Strawbridge $^{4}$. \\ Shenglan Yuan ${ }^{5}$
}

Published online: 17 June 2021

(c) Society for Mathematical Biology 2021

\section{Correction to: Bull Math Biol https://doi.org/10.1007/s11538-019-00648-3}

The original version of this article contains errors in the proofs of parts 5, 6, and 7 of Theorem 1 because Eqs. (14) and (15) are incorrect. In this correction we will provide proofs of parts 5 and 7 . We do not have a proof of part 6 , which will now be a conjecture supported by extensive numerical simulations.

Proof of parts 5 and 7 of Theorem 1: We will first prove boundedness. Since $\rho\left(A_{0}\right)<1$ and $\rho\left(A_{g(y)}\right)$ is a decreasing function of $y$ (by part 1 of Theorem 1), there exists $M_{1}>0$ and $\epsilon \in(0,1)$ such that $\rho\left(A_{g\left(M_{1}\right)}\right) \leq \epsilon$ so that if $B(t) \geq M_{1}$, then $p_{t} \leq g\left(M_{1}\right)$ and $\rho\left(A_{p_{t}}\right) \leq \epsilon$. Hence there exists $M$ such that for any $t_{1}, t \in \mathbb{Z}^{+}$with $t>t_{1}$,

The original article can be found online at https://doi.org/10.1007/s11538-019-00648-3.

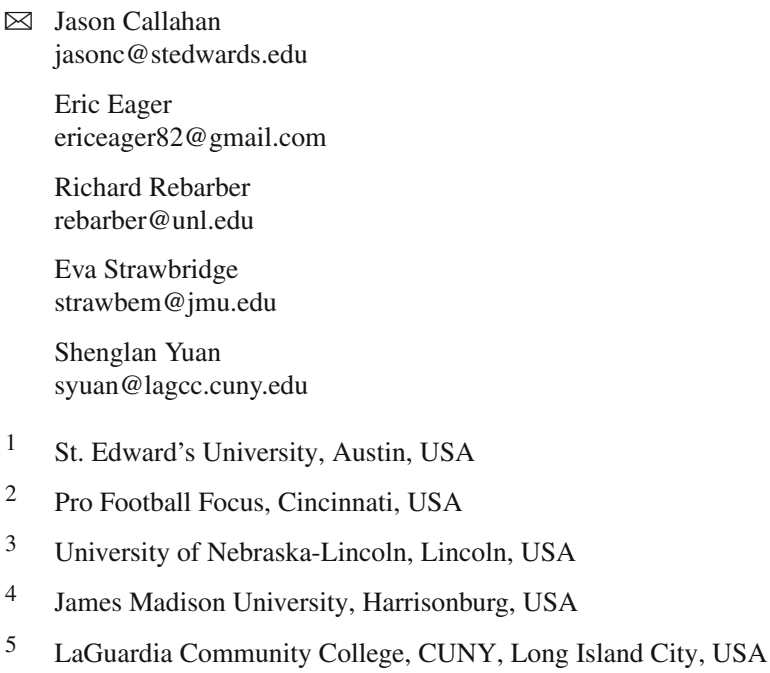




$$
\left\|A_{g\left(M_{1}\right)}^{t-t_{1}} \vec{P}\left(t_{1}\right)\right\| \leq M \epsilon^{t-t_{1}}\left\|\vec{P}\left(t_{1}\right)\right\| .
$$

Claim 1 There exists $M_{2}>0$ such that if $B(t)<M_{1}$, then $B(t+1)<M_{2}$.

Proof of Claim 1 If $B(t)<M_{1}$, then $\|P(t)\|<M_{1} / L_{0}^{3}$. We then use equation (1) from the original version of this article for $A_{p_{t}}$ to see that $\left\|A_{p_{t}}\right\| \leq s_{n}+f_{n}$. Hence

$$
B(t+1)=\vec{L}^{T} A_{p_{t}} \vec{P}(t) \leq \frac{L_{n}^{3} M_{1}}{L_{0}^{3}}\left(s_{n}+f_{n}\right),
$$

so we set $M_{2}=L_{n}^{3} M_{1}\left(s_{n}+f_{n}\right) / L_{0}^{3}$.

Now assume that $\|\vec{P}(0)\|$ is such that $B(0) \leq M_{1}$. Then either $B(t) \leq M_{1}$ for all $t>0$ (so $\vec{P}(t)$ is bounded by $M_{1} / L_{0}^{3}$ ) or there exists a largest $t_{1}$ such that $B(t) \leq M_{1}$ for $t=1,2, \ldots t_{1}-1$. By Claim $1, B\left(t_{1}\right)<M_{2}$, so $\left\|\vec{P}\left(t_{1}\right)\right\| \leq M_{2} / L_{0}^{3}$. Then for $t>t_{1}$ such that $B(\tau) \geq M_{1}$ for $\tau \in\left\{t_{1}, \ldots t\right\}$,

$$
\begin{aligned}
\|\vec{P}(t)\| & =\overrightarrow{1}^{T} \vec{P}(t)=\overrightarrow{1}^{T} A_{p_{t-1}} A_{p_{t-2}} \cdots A_{p_{t_{1}}} \vec{P}\left(t_{1}\right) \\
& \leq \overrightarrow{1}^{T} A_{g\left(M_{1}\right)}^{t-t_{1}} \vec{P}\left(t_{1}\right)=\left\|A_{g\left(M_{1}\right)}^{t-t_{1}} \vec{P}\left(t_{1}\right)\right\|
\end{aligned}
$$

using Lemmas 1 and 2. Hence for $t>t_{1}$ such that $B(\tau) \geq M_{1}$ for $\tau \in\left\{t_{1}, \ldots t\right\}$, using (1),

$$
\|\vec{P}(t)\| \leq \frac{M M_{2}}{L_{0}^{3}} \epsilon^{t-t_{1}} .
$$

The right side of (2) will decrease. If it decreases so much that $B\left(t_{2}\right)<M_{1}$ for some $t_{2}>t_{1}$, then we can start our argument again with time 0 replaced by time $t_{2}$. If it fails to decrease that much, then $\|\vec{P}(t)\| \leq M M_{2} / L_{0}^{3}$ for all $t>t_{1}$. Thus we see that if $\|\vec{P}(0)\|$ is such that $B(0) \leq M_{1}$, then

$$
\|\vec{P}(t)\| \leq \min \left\{M_{1}, M M_{2}\right\} / L_{0}^{3} .
$$

If $\|\vec{P}(0)\|$ is such that $B(0)>M_{1}$, then we can replace $M_{2}$ with $M_{3}=$ $\max \left\{M_{2}, B(0)\right\}$ from (2) on.

Remark 1 It follows from the proof of boundedness that the system is also point dissipative because for every initial condition, as $t \rightarrow \infty, B(t)$ eventually decreases below $M_{2}$, so $\|\vec{P}(t)\|$ eventually decreases below $\min \left\{M_{1}, M M_{2}\right\} / L_{0}^{3}$.

We now turn to uniform persistence and use Theorem 7.9 in Smith and Thieme (2011) to establish uniform convergence. We will show that the three hypotheses for this theorem are satisfied. Let

$$
F: \mathbb{R}^{n+1} \rightarrow \mathbb{R}^{n+1}, \quad F(\vec{x})=A_{g\left(\vec{L}^{T} \vec{x}\right)} \vec{x} .
$$


1. It is clear that $\mathbb{R}_{+}^{m} \backslash\{\overrightarrow{0}\}$ is forward invariant under $F$ since if $\vec{x} \in \mathbb{R}_{+}^{m} \backslash\{\overrightarrow{0}\}$, then $F(\vec{x}) \in \mathbb{R}_{+}^{m} \backslash\{\overrightarrow{0}\}$. Hence hypothesis (a) of Theorem 7.9 in Smith and Thieme (2011) is satisfied.

2. We first compute the Jacobian of $F$ at the origin, denoted by $J(\overrightarrow{0})$. It is easy to check that

$$
J(\overrightarrow{0})=A_{1} .
$$

By the hypotheses of part 5 of Theorem $1, \rho(J(\overrightarrow{0}))=\rho\left(A_{1}\right)>1$. Let $\vec{\phi}$ be an eigenvector of $A_{1}^{*}$ (the adjoint of $A_{1}$ ) associated with $\rho\left(A_{1}^{*}\right)=\rho\left(A_{1}\right)$, which is guaranteed to be strictly positive by the Perron-Frobenius Theorem. Since $A_{1} \vec{\phi}=$ $\rho\left(A_{1}\right) \vec{\phi}$, hypothesis (b) of Theorem 7.9 in Smith and Thieme (2011) is satisfied.

3. This hypothesis is that the system is point dissipative, which we established above; see Remark 1.

Theorem 7.9 in Smith and Thieme (2011) then gives that the system is uniformly persistent.

Remark 2 In the statement of Theorem 2, $B^{*}$ is identified as the "limiting biomass." Since the proof of part 6 is incorrect, that identification is no longer true. If the words "limiting biomass" are removed, the statement of Theorem 2 and its proof are still true.

We were not able to prove part 6 of Theorem 1 , which states that when $\rho\left(A_{0}\right)<1<$ $\rho\left(A_{1}\right)$ the biomass converges, but we can support this numerically as follows. We ran numerical simulations for ten thousand randomly selected parameter sets consisting of $\vec{s}, \vec{f}$, and $\vec{P}_{0}$ with 'convergence' defined as the last five time step values of the biomass all being within $10^{-2}$ of each other. Both $\vec{s}$ and $\vec{f}$ were required to be increasing, positive vectors with $0<s_{n}<1$. A condition on $\vec{f}$ to ensure $\rho\left(A_{0}\right)<1$ is that $f_{1}<\left(1-s_{1}\right) / s_{0}$, but if $f_{1}$ is close to this value, $\rho\left(A_{0}\right)$ is close to one and numerical convergence takes a long time. For the purposes of our simulations we forced $f_{1}$ to be randomly selected between zero and ten percent of this upper limit. The fecundities in $\vec{f}$ do not have a natural upper bound, so we chose an arbitrary upper bound of 25 . Similarly, the initial population vector $\vec{P}_{0}$ does not have a natural upper bound, but each entry was selected randomly and independently between 0 and 100 with entries not required to be increasing. In every case we ran, the biomass converged in the sense above, giving credence to the result in part 6 in the absence of a proof.

\section{References}

Smith HL, Thieme HR (2011) Dynamical systems and population persistence. American Mathematical Society, Providence

Publisher's Note Springer Nature remains neutral with regard to jurisdictional claims in published maps and institutional affiliations. 